\title{
Superior oblique tendon sheath syndrome and its relationship to stenosing tenosynovitis
}

\author{
J. H. SANDFORD-SMITH \\ Bristol Eye Hospital
}

The superior oblique tendon sheath syndrome, first describes! by Brown (1950), still attracts some interest because the aetiology remains obscure and treatment and management are subject to controversy.

Various theories have been proposed concerning the nature of the abnormality:

(r) The main feature of the syndrome is the thickened sheath of the superior oblique tendon, and it is thought that this mechanically prevents separation of the insertion of the superior oblique tendon from the trochlea, and hence produces the clinical manifestations of the syndrome (Brown, i 950, I 957).

(2) Electromyographic recordings from the superior oblique and inferior oblique muscles have suggested that the condition is due to abnormal innervation of these muscles (Papst and Stein, I969).

(3) An isolated inferior oblique paralysis bears certain clinical similarities to the superior oblique tendon sheath syndrome and it has been proposed that this is the underlying cause of the thickencd superior oblique tendon sheath which acts as the check ligament of the inferior oblique (Brown, I 957).

(4) As an attempt to explain specifically the intermittent cases, those associated with a clicking sound or sensation, and those showing spontaneous resolution, it has been suggested that there is a circumferential constriction of the trochlea and sheath preventing a locally enlarged tendon from sliding freely in the sheath. This concept, first proposed by Girard (1956), has been mentioned by other authors (Brown, I962b; Goldstein, r969; Sandford-Smith, I969; Catford and Dean Hart, I97I). The marked clinical similarity of the tendon sheath syndrome to the common orthopaedic condition of stenosing tenosynovitis was first noted by Sandford-Smith ( 1969 ) and it was suggested that this may be the underlying pathological charge, not only in the intermittent cases, but in the persistent ones.

Mein (1971), in a recent review of 37 cases, considered stenosing tenosynovitis to be the most likely cause of the tendon sheath syndrome.

Hitherto, the evidence for this concept has been circumstantial, but two patients are described below which give more direct evidence for this theory that the tendon sheath syndrome is a type of stenosing tenosynovitis.

A third case is also described in which an attempt was made to carry out surgical treatment similar to that used for stenosing tenosynovitis in other parts of the body. 


\section{Case reports}

Case I, a 3-year-old girl, presented with a suspected squint.

\section{Examination}

There was intermittent left hypotropia in the primary position with a marked depression of the left eye in adduction, and an intermittent esotropia, especially for near. Atropine refraction and $\overline{\frac{O}{9}}$ fundus examination were normal.

A provisional diagnosis of a left superior oblique tendon sheath syndrome was made which was confirmed by a duction test under anaesthesia.

\section{Operation}

As binocularity was threatened, and the cosmetic appearance was not satisfactory, the sheath was explored through a conjunctival incision and found to be thickened. During exposure, while․ㅡㅇ. traction was applied to the tendon and sheath, a sudden "release" was felt and a duction test then showed a full range of passive movement. After the sheath had been incised longitudinally, one $-\overrightarrow{-}$ thickened segment of the sheath was found to be particularly swollen and constricted circumferen-io tially, and on the ocular side of this constriction there was a fusiform dilatation of the tendon. It seemed reasonable to assume that the dilated tendon had initially been unable to pass through the $\frac{}{5}$ constricted sheath on passive stretching, and that the manipulation of exposure of the sheath enabled this constriction to be overcome.

It was also noted that the tendon was inserted on the medial side of the superior rectus, thus increasing the trochlear angle. After longitudinal incision, but not excision, of the sheath, the tendon moved freely in the sheath. Io mg. Depo-Medrone (a depot preparation of dexamethasone) were injected into the tissues around the sheath and the conjuctival incision was closed.

\section{Result}

Up to 3 months post-operatively, ocular movements were greatly improved, with only a minimal restriction of the globe in extreme dextro-elevation, and the secondary over-convergence was spon-o taneously controlled. However, after a further 2 months, marked limitation of elevation in adduc-气ू tion was again apparent.

Case 2, an 8-year-old girl, presented with defective elevation of the right eye. On questioning, her mother thought that the eye movements had probably been restricted ever since early childhood.? There was no history of trauma.

\section{Examination}

There was limitation of movement of the right eye in laevo-elevation. A nodule about $5 \mathrm{~mm}$. in diameter could be palpated behind the trochlea. A Hess chart (Fig. I) showed the typical changes $\delta$ of the superior oblique tendon sheath syndrome, but, as the symptoms were minimal and full binocular vision was present, no treatment was suggested.

\section{Progress}

After 3 years the situation was virtually unchanged, except that the nodule had perhaps become â little larger. On laevo-depression the nodule tended to recede, and on attempted laevo-elevation it $N$ became more prominent. It was therefore, presumably, situated in the superior oblique tendon and స్ట was lying posterior (on the muscle side) to the trochlea (Fig. 2).

Case 3, a I-year-old girl, presented with a suspected squint.

\section{Examination}

There was a left convergent squint with defective elevation of the left eye in adduction. Ductionsö under anaesthetic confirmed the inability of the left eye to elevate passively in adduction, and ao left superior oblique tendon sheath syndrome was diagnosed. 

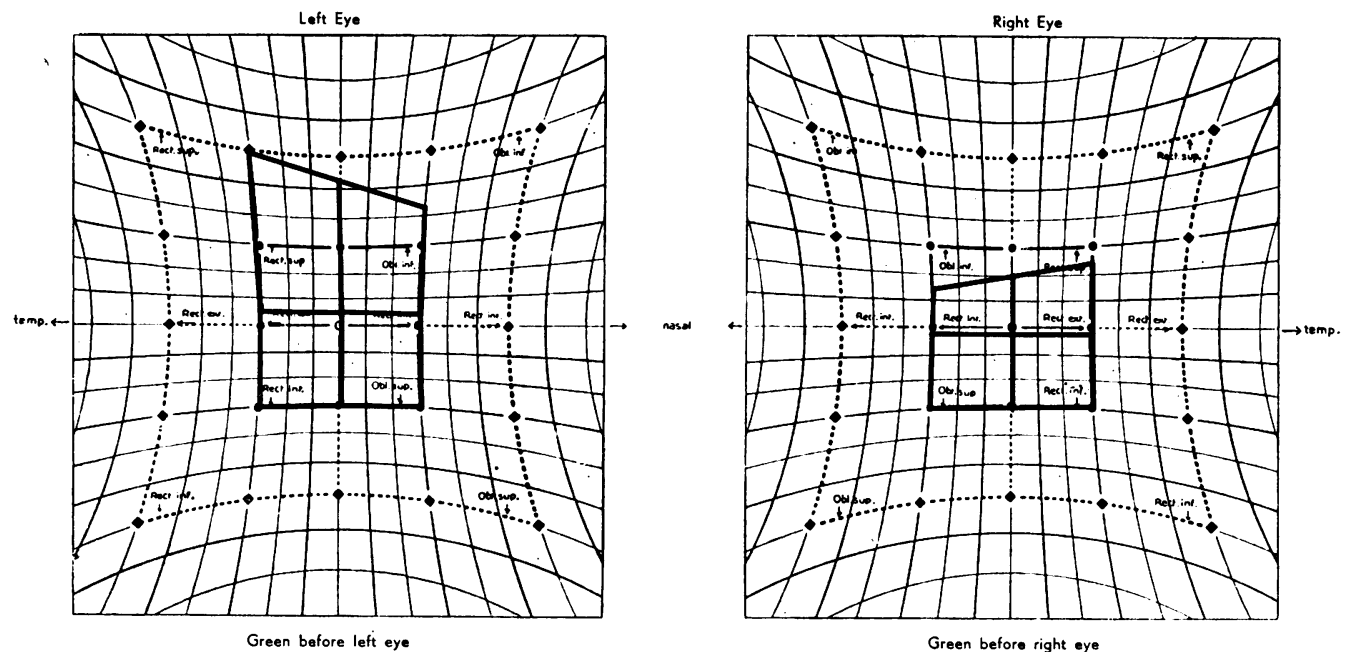

FIG. I Case 2. Hess chart, showing restricted eye movements typical of the superior oblique tendon sheath syndrome
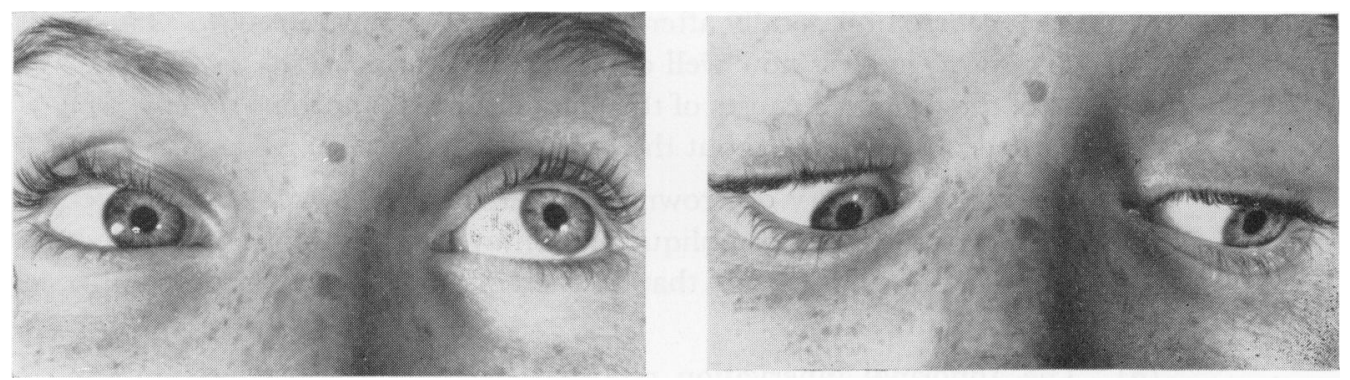

FIG. 2 Case 2. Laevo-elevation and laevo-depression, showing a nodule at the trochlea on attempted laevoelevation receding into the orbit on laevo-depression

\section{Progress}

After I year the situation had not altered and as there was some left hypotropia in the primary position, surgical treatment was suggested.

\section{Operation}

On exploration the sheath was found to be slightly thickened but when it was excised longitudinally, the underlying tendon was normal and duction tests remained positive. Therefore, the trochlea itself was also incised with scissors, whereupon ductions immediately became full and the fascial connections around the trochlea seemed to prevent any significant bow-stringing of the tendon which would cause a spurious increase in ductions. Unfortunately, the exposure was not adequate to verify whether or not there was any thickening of the tendon at the trochlea.

\section{Result}

Immediately post-operatively, the child's condition greatly improved, with full elevation of the affected eye, but 2 weeks later, when she was seen again, some restriction of elevation in adduction was again apparent but less than pre-operatively. 
Second operation

After a further 2 weeks, the condition had deteriorated to the pre-operative state and, as further $\frac{0}{3}$

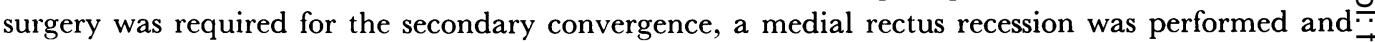
the superior oblique tendon was re-examined. Numerous adhesions were seen between the sheath $\stackrel{\vec{F}}{\vec{F}}$ and the tendon, increasing towards the trochlea. Presumably, the trochlear mechanism and theo synovial sheath are too delicate to survive extensive surgical interference without adhesions.

\section{Discussion}

The superior oblique tendon sheath syndrome may comprise a group of conditions of $\overrightarrow{0}$ varying nature with a common clinical presentation (Girard, r956), but the physical signs are so specific that it seems reasonable to postulate a single aetiological factor. In $\vec{\sigma}$ particular, it may be thought that there are basically two types:

(I) The less common intermittent cases which are often associated with clicking sen-o sations, and an improvement after attempted forced movements or manipulation over the trochlea;

(2) The more common "persistent" cases.

However, there appears to be no clear distinction between the two types as is exemplifiedby a patient who showed the characteristic features of the tendon sheath syndrome whicho spontaneously remitted after 13 years (Brown, I962a); or a second case in which gradual@ remission occurred bilaterally after at least 6 years (Lowe, i 969 ). The tendency to spon-.

taneous improvement is now well documented (Mein, I97I).
Some of the proposed causes of the tendon sheath syndrome do not easily account for the recorded observations about the syndrome:

(I) The original theory of Brown (i.e. that the thickened sheath prevents movement the inertsion of the superior oblique tendon away from the trochlea) does not explain theo intermittent cases, and it seems that excision of the sheath alone does not generally produceٍ satisfactory results.

(2) The abnormal innervation reported by some authors has not been confirmed by others who found normal electromyographic recordings of both the superior and inferior oblique muscles (Catford and Dean Hart, I97I). Also, such a theory neither explains how a forced duction test under anaesthesia could show passive resistance to movement $\frac{0}{0}$. nor accounts for the intermittent cases.

(3) The concept of a true congenital inferior oblique palsy producing a contracture of itsio check ligament, the sheath of the superior oblique, would explain the often poor results oß excision of the sheath; but it could not account for the inferior oblique overaction that has been reported after superior oblique tenotomy (Haworth, 1970), and does not explain the intermittent cases; it also appears to be unsupported by electromyography (Breinin, 1957)

In addition, the characteristic sequelae of vertical muscle palsies towards concomitance are not usually seen.

All the phenomena of the superior oblique tendon sheath syndrome can be satisfactorily explained according to the last theory-namely, that the trochlea and sheath form a constriction preventing a slightly swollen tendon from entering the trochlea and the sheath Wherever a tendon alters its direction of action acutely, there must be a tight restraining sheath, and in such a situation a swelling of the tendon associated with constrictive hyper-T trophy of the sheath is quite a common pathological change and is known as stenosing tenosynovitis or tenovaginitis (tenosynovitis stenosans). 
It is logical to assume that tendons and their sheaths with similar anatomical structures should undergo similar pathological changes; and in a previous article the author has elaborated on the anatomical and clinical similarities of stenosing tenosynovitis and the tendon sheath syndrome (Sandford-Smith, i 969 ).

According to this theory, the cardinal symptom and sign of the tendon sheath syndrome - the inability of the eye to elevate in adduction, either actively or passively in a forced duction test, is caused by the inability of the locally hypertrophied tendon to enter the trochlea sheath complex, but there is no restriction to contraction of the superior oblique as there is no tight sheath posterior to the trochlea.

The intermittent and permanent cases are essentially the same in nature, but in the former the swollen tendon is sometimes able to pass through the trochlea and sheath, often with a click or with delay or with only an effort; in the latter, on the other hand, the tendon is not able to pass through the sheath.

Theoretically, it may be possible for the swollen tendon to pass into the sheath but not or only with difficulty out of it again on contraction of the superior oblique. This would produce a variable and intermittent superior oblique weakness associated with clicking and such a condition has indeed been recently described (Foster, 1970).

Presumably, the narrowest part of the trochlea and of the sheath, which is in direct continuity with it, is in most cases the trochlea itself, and so one would not expect mere excision of the sheath to be generally successful in relieving the condition.

Similarly, a tenotomy or tendon lengthening of the superior oblique by allowing a greater range of passive stretching of the tendon before the enlarged segment abuts against the trochlea, would be expected to alleviate the condition, although at the expense of weakening the action of the superior oblique.

Both these therapeutic concepts seem to be borne out by experience.

The basis of the condition appears to be mechanical and is probably caused by wear and tear changes where the tendon alters its direction sharply at the trochlea. This would explain the traumatic cases in which any injury at the trochlea is likely to harm the delicate synovial sheath and thus provide a basis for wear and tear changes.

One slightly unsatisfactory aspect of this theory is that excision of the sheathalthough apparently rarely producing full ocular movements-is reported as frequently improving passive ductions at the time of operation, and also as alleviating the widening of the palpebral fissure and the pulling sensation experienced by the patient on attempted elevation in adduction. A possible explanation is that the sheath undergoes fibrosis and contracts not only in a circumferential direction but in a longitudinal one as well. Alternatively, it has been suggested that division of the sheath allows the superior oblique tendon to retract slightly (Stanworth, I97I). Also, the observation that there was no restriction to passive stretching of the superior oblique tendon although there was restriction to stretching of the sheath (Brown, I957) would refute this theory but the author has been unable to confirm this observation.

It is also interesting that in Case I there was an abnormal superior oblique insertion on the globe which would increase the acuteness of the trochlear angle and predispose to secondary changes in the tendon and sheath. (A similar abnormal insertion has also been noted on another occasion by the author, in a case treated by superior oblique tenotomy).

Such an anatomical variant could be an inherited factor which might explain the familial incidence of the disorder which has been reported (Gowan and Levy, i968). It is reason- 
able to suppose that it may be bilateral and, may hence, be a factor in the frequently reported bilateral cases.

In the first two cases here described, direct evidence is produced to show that the tendon sheath syndrome is a type of stenosing tenosynovitis. In the first case, a localized dilat- $\stackrel{\bar{\Xi}}{\stackrel{D}{7}}$ ation of the tendon was observed, associated with thickening, circumferential stenosis, 등 and some oedema of the sheath. These are the changes found in stenosing tenosynovitis. Whenever the superior oblique tendon sheath is explored, only the thickened sheath and not the dilated tendon is usually observed - this is to be expected as, presumably, the place of greatest stenosis is usually the trochlea itself and so any dilatation of the tendon would usually be located posterior to it and hence not seen on exploration. This case was perhaps unusual in that the most constricted area was apparently in the sheath between the trochlea and the globe.

In the second case an unusually large dilatation of the tendon, posterior to the trochlea, could be felt through the skin.

In the third case, as an attempt further to prove the validity of these concepts, the entire sheath, up to and including the trochlea, was incised longitudinally to allow free passage of of the swollen tendon through it. This is analogous to the surgical treatment of stenosing tenosynovitis in the hand.

The good initial result might be taken as confirmation of the hypothesis, but the rapid $z$ onset of fibrosis and adhesions between the tendon and sheath confirms the findings noted by others that surgery of the sheath may cause post-operative adhesions between the tendon and its sheath (Scott and Knapp, 1972). Surgery of the trochlea is generallo considered to be unsatisfactory (Hugonnier and Clayette-Hugonnier, r969), and the attempted treatment in Case 3 would confirm this although it is possible that a dire approach to the trochlea through the skin may be less traumatic. Transposition of the्e trochlea has been apparently successful by this route, and so presumably an incision of it may be feasible (Hughes and Bogart, 1942).

Perhaps further research into the possibility of incising the superior oblique sheath and the trochlea may be of value, and the injection of "depot" preparations of steroids may lessen the tendency to the development of fibrosis post-operatively: at present, however, tenotomy of the superior oblique seems to be the most effective type of treatment should surgery be considered necessary. Such an operation, however, is not ideal because of the risk of losing binocularity in the direction of action of the tenotomized superior oblique, or the possibility of a secondary overaction of the inferior oblique.

Thus, the concept of stenosing tenosynovitis helps to explain many of the puzzling features of the superior oblique tendon sheath syndrome, but unfortunately these ideas have not yet led to any marked improvement in the treatment of this condition.

\section{Summary}

Three cases of the superior oblique tendon sheath syndrome are described. The various $\mathcal{N}$ theories about the underlying pathological changes in this syndrome are discussed and it is $N$ shown that the concept of stenosing tenosynovitis-i.e. a hypertrophy and constriction of $\mathbb{W}^{N}$ the trochlea and tendon sheath associated with localized swelling of the tendon-best $\mathcal{F}_{2}$ explains the observed phenomena of the syndrome. The cases presented show some direct $\stackrel{\varrho}{\mathbb{C}}$ evidence for this theory. Some suggestions are made as to possible methods of treatment.

I wish to record my thanks to Mr. C. A. Brown and Mr. V. J. Marmion for permission to examine and treat patients under their care, to Mr. J. C. Dean Hart for advice in the preparation of this paper, and to the staff of the Orthoptic Department, Bristol Eye Hospital, for their interest in and assessment of the patients described. 


\section{References}

Breinin, G. м. (1957) A.M.A. Arch. Ophthal., 58, 375

BRown, H. W. (1950) In "Strabismus: Ophthalmic Symposium (I)", ed. J. H. Allen, p. 205. Mosby, St. Louis

(1957) Trans. Amer. ophthal. Soc., 55, 415

(1962a) In "Strabismus, Symposium of the New Orleans Academy of Ophthalmology", ed. G. M. Haik, p. 252. Mosby, St. Louis

$$
\text { ( } 1962 \text { b) Idem, p. } 345
$$

CATFORD, G. v., and DeAN HART, J. c. (I97I) Brit. F. Ophthal., 55, I 55

FOSTER, s. (1970) Brit. orthopt. F., 27, 125

Girard, L. J. (1956) Sth. med. F. (Bgham, Ala.), 49, 342

goldstein, J. H. (1969) Amer. F. Ophthal., 67, 96o

GOWAN, M., and LEVY, J. (1968) Brit. orthopt. F., 25, 9 I

HAWORTH, s. M. (1970) Brit. J. Ophthal., 54, 675

hUGHes, W. L., and Bogart, D. w. (1942) Amer. F. Ophthal., 25, 9 I I

HUGONNIER, R., and ClAyetTe-HUgonNier, s. (1969) In "Strabismus, Heterophoria, Ocular Motor

Paralysis", trans. from 2nd French ed. by S. Veronneau-Troutman, p. 6I5. Mosby, St. Louis LOWE, R. F. (1969) Brit. 7. Ophthal., 53, 466

MEIN, J. (I97I) Brit. orthopt. F., 28, 70

papst, w., and stein, H. J. (1969) Klin. Mbl. Augenheilk, 154, 506

SANDFORD-SMITH, J. H. (1969) Brit. F. Ophthal., 53, 4 I 2

STANWORTH, A. (197I) Brit. orthopt. F., 28, 23

sCotT, A. B., and KNAPP, P. (I972) Arch. Ophthal. (Chicago), 88, 282 